\title{
Immune responses in DNA vaccine formulated with PMMA following immunization and after challenge with Leishmania major
}

\author{
Somayeh Zarrati $\cdot$ Mehdi Mahdavi • \\ Fatemeh Tabatabaie
}

Received: 20 April 2014/ Accepted: 8 August 2014/Published online: 31 August 2014

(C) Indian Society for Parasitology 2014

\begin{abstract}
Leishmaniasis is a major infectious disease caused by protozoan parasites of the genus Leishmania. Despite of many efforts toward vaccine against Leishmania no effective vaccine has been approved yet. DNA vaccines can generate more powerful and broad immune responses than conventional vaccines. In order to increase immunity, the DNA vaccine has been supplemented with adjuvant. In this study a new nano-vaccine containing TSA recombinant plasmid and poly(methylmethacrylate) nanoparticles (act as adjuvant) was designed and its immunogenicity tested on BALB/c mouse. After three intramuscular injection of nano-vaccine $(100 \mu \mathrm{g})$, the recombinant TSA protein $(20 \mu \mathrm{g})$ was injected subcutaneously. Finally as a challenge animals were infected by Leishmania major. After the last injection of nano-vaccine, after protein booster injection, and also after challenge, cellular immune and antibody responses were evaluated by ELISA method. The findings of this study showed the new nano-vaccine was capable of induction both cytokines secretion and specific antibody responses, but predominant Th1 immune response characterized by IFN- $\gamma$ production compared to control groups. Moreover, results revealed that nano-vaccine was effective in reducing parasite burden in the spleen of Leishmania major-infected BALB/c mice. Base on
\end{abstract}

\footnotetext{
S. Zarrati

Microbiology Department, Science \& Research Branch, Islamic Azad University, Tehran, Iran

M. Mahdavi

Virology Department, Pasteur Institute of Iran, Tehran, Iran

F. Tabatabaie $(\bowtie)$

Department of Parasitology and Mycology, Faculty of Medicine, Iran University of Medical Sciences (IUMS), Tehran, Iran e-mail: dr.f.taba@hotmail.com; tabatabaei.f@iums.ac.ir
}

results, current candidate vaccine has potency for further studies.

Keywords TSA - Leishmania major .

Poly(methylmethacrylate) · Immune response

\section{Introduction}

Leishmaniasis, caused by an intracellular protozoan parasite, is widespread in many parts of the world with about 12 millions infected cases. There are 1.5-2 millions of new cases of cutaneous leishmaniasis and 500,000 cases of visceral leishmaniasis reported annually. Infection with HIV/AIDS can increase the risk of developing leishmaniasis by 100-1,000 folds (Webb et al. 1998; Mendez et al. 2002; Campos-Neto et al. 2002). Chemotherapeutics are available but show high toxicity, costs and are prone to resistance development due to prolonged treatment period. So many studies have been developed to prepare a protective vaccine. Development of either new anti-Leishmania drugs or a vaccine is an attractive alternative. Immunity against reinfection is acquired following cutaneous infection with Leishmania spp., suggesting that prophylactic immunization is feasible (Gradoni 2001; Campos-Neto et al. 2001; Handman 2001). A number of Leishmania vaccine candidates, including killed parasites, crude parasite fractions, recombinant Leishmania antigens, and antigen-encoding DNA, have been investigated in murine models. But in spite of several tested vaccine protocols no protective vaccine against any clinical leishmaniasis has been produced commercially (Saldarriaga et al. 2006; Ahmed et al. 2009). DNA vaccination is a new immunization strategy that has many potential advantages over other vaccine strategies. The major 
advantage of DNA vaccine is induction the expression of antigens, which are unaltered in their protein structure and antigenicity (Zadeh-Vakili et al. 2004; Sambrook et al. 1989).Most of the works have focused on different antigens Among the vaccine candidates, TSA (Thiol-Specific Antioxidant protein) has been introduced as one of the predominant vaccine candidates. TSA is $\mathrm{L}$ major recombinant protein homologue to eukaryotic Thiol-SpecificAntioxidant protein with molecular weight of $22.1 \mathrm{KDa}$ is composed of 200 amino acids and placed in the chromosome of 15. TSA is expressed in L. major promastigote and amastigote (Rafati et al. 2006a, b; Mendez et al. 2001; Monnerat et al. 2004). TSA DNA vaccine stimulated high titers of specific IgG2a antibody, high levels of IFN- $\gamma$ and low levels of IL-4, phenotypic markers of Th1 responses, which are the type of immune responses required for the control of this parasite. Many efforts to develop effective Leishmania vaccine have been limited due to lack of an appropriate adjuvant. Nanoparticles are solid very small fragment ranging in size from 1 to $1,000 \mathrm{~nm}(1 \mu \mathrm{m})$. They consist of macromolecular materials and can be used therapeutically or prophylactically, for example, as an adjuvant in vaccines or drug carriers, in which the active principle is dissolved, drew or encapsulated, or to which the active principle is adsorbed or chemically attached. Nanoparticles are able to enter antigen-presenting cells by different pathways, thereby regulating the immune response to the antigen. Their properties also make them appropriate for the delivery of antigens at mucosal surfaces and for intradermal administration. It is generally agreed that the adjuvanticity of nanoparticles and microparticles is affected by particle sizes, which in turn affect the type of immune responses caused by antigens carried by particles. Particulate carriers can serve as an effective antigen delivery system and, thus, improve and/or facilitate the uptake of antigens by antigen-presenting cells such as dendritic cells or macrophages. Particle-based antigen carriers may attend as a depot for controlled release of antigen, thereby increasing the availability of antigens to the immune cells. Poly(methylmethacrylate) (PMMA) is a synthetic polymer approved by the Food and Drug Administration for specific human clinical applications such as the bone cement. In vivo, PMMA particles are phagocytosable and have the potential to initiate strong immune responses by stimulating the production of inflammatory cytokines (Mutiso et al. 2010; Lou et al. 2009; O'Hagan 2000; Stieneker et al. 1995) The purpose of this work was DNA-vaccine efficacy in the presence PMMA adjuvant comparing to absence of it. We evaluated the usefulness of PMMA as a nano-adjuvant with DNA vaccine encoding TSA antigen of L. major in BALB/c mice in order to optimize the efficacy of the vaccine against leishmaniasis.

\section{Methods}

\section{L. major promastigotes}

The MHRO/IR/75/ER (an Iranian strain to be isolated by Nadim et al. in 1964) of L. major was provided by Pasteur Institute of Iran. Promastigotes were grown at $26{ }^{\circ} \mathrm{C}$ in RPMI1640 medium (Sigma ${ }^{\circledR}$ ) supplemented with $10 \%$ heat inactivated fetal calf serum $\left(\mathrm{Gibco}^{\circledR}, \mathrm{BRL}\right)$, and $100 \mu \mathrm{g} / \mathrm{ml}$ gentamicin $\left(\operatorname{Sigma}^{\circledR}\right)$. Stationary phase of the promastigotes were harvested at a density of $1 \times 10^{6} / \mathrm{ml}$.

Plasmid constructions

The TSA recombinant plasmid DNA was prepared in a previous study (Tabatabaie et al. 2007) transformed into E. coli DH5- $\alpha$ and purified by plasmid extraction Kit (Bioneer, Germany), dissolved in sterile deionizer distilled water and stored at $-20{ }^{\circ} \mathrm{C}$ until use. Then the EndoFree plasmid purification Giga Kit (Qiagen, CA, USA) was used according to the manufacturer's instructions. DNA concentrations were measured by absorbance at $260 \mathrm{~nm}$. The OD260/280 ratios for the purified DNA were 1.80-1.95, indicating that the preparations were free from protein contamination.

Preparation of vaccine

The PMMA polymeric nanoparticles used as adjuvant were produced by gamma irradiation polymerization method in the absence of antigen (Kreuter 1995; O'Hagan 2000). In order to prepare the nano-vaccine candidate, pcDNA3/TSA recombinant plasmid was loaded to PMMA nanoparticles. In brief, 10 Mm EDAC 1-ethyl-3-(3-dimethylaminopropyl) carbodiimide (EDAC) was added to $1 \mathrm{Mm}$ of PMMA nanoparticles solution and incubated for $10 \mathrm{~min}$ at room temperature with gentle stirring. After that, $1 \mathrm{ml}$ Plasmid DNA solution of $100 \mu \mathrm{g} / \mathrm{ml}$ was added (equal volumes of the two solution were mixed) and then left in the cold room overnight. For purification, the solution was further subjected to extensive dialysis. The resulting PMMA-plasmid DNA nanoparticles were preserved in suspension form in double-distilled water. Size of nanoparticles were determined using a Zeta Sizer (Malvern, UK) (data not shown). The TSA recombinant peptide booster $(22 \mathrm{KD})$ was gifted by Miss Narges Khabbaz zade Tehrani from Faculty of Basic Sciences, Science and Research Branch of Islamic Azad University of Tehran.

Immunization and experimental infection of the mice

Inbred Female BALB/c (6-8 weeks old) mice were purchased from Lab. Animal Center (Pasteur Institute, Karaj, 
Iran) and handled in accordance with the National Animal Care and Use protocol at Iran University of Medical Sciences. Mice were divided into two test (T) and three control (C) groups (20 mice/group) and respectively received DNA Vaccine (pcDNA3/TSA), nano-vaccine (pcDNA3/ TSA + PMMA) and pcDNA3 (as control group) in dose of $100 \mu \mathrm{g}$. Another control groups were injected with PMMA and PBS. The mice in each group were anesthetized with $25 \mu \mathrm{g} \mathrm{g}^{-1}$ of mixture of ketamine $10 \%$ and xylazine $2 \%$ via intraperitoneal (i.p.). All injections were done intramuscularly (i.m.) and the injection sites were immediately pulsed using tweezer-type electrodes (CUY650, Sonidel Limited $^{\circledR}$, Ireland) to administer eight $60 \mathrm{~V}$ pulses each of $20 \mathrm{~ms}$ duration with a $200 \mathrm{~ms}$ interval using a BTX ECM830 generator (Harvard Apparatus, USA). The mice were immunized (i.m.) injection into both quadriceps's with $100 \mu \mathrm{l}$ of PBS, $50 \mu \mathrm{l}$ in each anterior tibialis muscle (Ahmed et al. 2009). Three inoculations were employed with the same DNA and PMMA doses and the same immunization schedule was applied at 3 weeks interval. Two weeks after the last injection of nano-vaccines, $20 \mu \mathrm{g}$ booster peptide plus incomplete Freund's adjuvant was injected subcutaneously. Three weeks after final immunization mice were challenged with $1 \times 106$ promastigotes of L. major (strain MHRO/IR/75/ER) at the base of tail by the intradermal route (Sasaki et al. 2003). Two weeks after the last injection of nano-vaccine, two weeks after the peptide booster injection and five weeks after parasite challenge the animals were sacrificed and the spleens and serum samples were harvested for immunological analysis.

\section{Lymphocyte proliferation assay}

Spleen was taken from each individual mouse, dissected and resuspended in sterile cold PBS containing $2 \%$ FBS. RBCs were lysed with lysis buffer, and single-cell suspension was adjusted to $3 \times 106$ cells/ml in RPMI 1640 (Gibco, Germany) supplemented with $10 \% \mathrm{FBS}, 4 \mathrm{mM}$ L-glutamine, $1 \mathrm{mM}$ sodium pyruvate, $50 \mu \mathrm{m} 2 \mathrm{ME}, 100 \mu \mathrm{g} / \mathrm{ml}$ streptomycin and $100 \mathrm{IU} / \mathrm{ml}$ penicillin. One hundred $\mu \mathrm{l}$ of the cell suspension was dispensed into 96-well flat-bottom culture plates and stimulated with $10 \mu \mathrm{g} / \mathrm{ml}$ of recombinant TSA protein (expressed in E. coli cells) as antigen recall. Phytohemagglutinin-A ( $5 \mu \mathrm{g} / \mathrm{ml}$, Gibco) was used as a positive control. Un-stimulated wells were used as the negative controls and complete culture medium was used as blank. All experiments were done in triplicate. After $72 \mathrm{~h}$ of culture, $100 \mu \mathrm{l}$ of 5-bromo-2-deoxy-uridine (Brdu) labeling solution was added into each well and incubation continued for $18 \mathrm{~h}$. The plates were then centrifuged and after removing culture medium wells were dried and fixed with $100 \mu \mathrm{l}$ of fixation/permeabilization buffer. Subsequently, $100 \mu l$ of anti-Brdu antibody was added to each well and the plates were washed four times and tetramethylbenzidine (TMB) substrate was added. The reaction was stopped by adding $100 \mu \mathrm{l}$ of $2 \mathrm{~N} \mathrm{H}_{2} \mathrm{SO}_{4}$. Optical density (OD) for each well was measured at $450 \mathrm{~nm}$. Stimulation Index (SI) was calculated according to the formula: OD of stimulated wells/OD of un-stimulated wells.

Cytokines assay before and after the challenge infection with L. major

A total number of $3 \times 106$ cells from single-cell suspension of each mouse spleen were placed onto the wells of a 24-well plate, stimulated in vitro with $10 \mu \mathrm{g} / \mathrm{ml}$ of recombinant TSA protein and incubated at $37{ }^{\circ} \mathrm{C}$ in $5 \%$ $\mathrm{CO}_{2}$. Seventy two hrs post antigen recall, supernatants were collected and centrifuged at $300 \times g$ for $10 \mathrm{~min}$ and stored at $-70{ }^{\circ} \mathrm{C}$ for cytokine analysis. IFN- $\gamma$ and IL-4 cytokines were quantified using commercial ELISA Kits (Mabtech sweden) according to the manufacturer's instructions. The quantity of each cytokine was reported as $\mathrm{pg} / \mathrm{ml}$ according to the plotted standard curve.

ELISA of total antibodies and IgG1, IgG2a subclasses

To evaluate the humoral immune responses, before and after challenge with L. major, sera of experimental groups were collected and specific antibodies were determined by an optimized indirect ELISA method. Briefly, $100 \mu \mathrm{l}$ of $10 \mu \mathrm{g} /$ $\mathrm{ml}$ of antigen in PBS buffer were added into 96-well ELISA Maxisorp plates (Nunc, Naperville, IL) and incubated $24 \mathrm{~h}$ at $37{ }^{\circ} \mathrm{C}$. The wells washed with PBS containing $0.05 \%$ Tween 20 (washing buffer) and blocked $1 \mathrm{~h}$ at $37^{\circ} \mathrm{C}$ with $5 \%$ skimmed milk in PBS (blocking buffer). Plates were washed with washing buffer and $100 \mu \mathrm{l}$ of $1 / 100$ diluted sera were added to each wells and incubated at $37{ }^{\circ} \mathrm{C}$ for $2 \mathrm{~h}$. The wells washed five times with washing buffer and incubated for $2 \mathrm{~h}$ with $100 \mu \mathrm{l}$ of 1/7,000 dilution of anti mouse conjugated to HRP (Sigma, USA). The wells washed five times and incubated 30 min with $100 \mu \mathrm{l}$ of TMB substrate in the dark and reaction was stopped with $2 \mathrm{~N} \mathrm{H} 2 \mathrm{SO} 4$ and color density was measured at A $450 \mathrm{~nm}$ with ELISA plate reader. Specific IgG1 and IgG2a subclasses were detected using goat anti mouse IgG1 and IgG2a secondary antibodies (Sigma, USA) according to the manufacture's instruction.

Determination of parasite burden

Three mice from each group were sacrificed and parasite burden draining spleens was determined using the limiting dilution method (Ahmed et al. 2009). Briefly, seven weeks after challenge a piece of spleen was excised, weighed and then homogenized with a tissue grinder in $2 \mathrm{ml}$ of RPMI 1640 medium (Gibco, Germany) supplemented with $20 \%$ heatinactivated fetal calf serum and Gentamicin $(0.1 \%)$.Under 
sterile conditions, the serial dilutions were prepared in wells of 96 well micro titration plates. After 7 days of incubation at $26{ }^{\circ} \mathrm{C}$, the plates were examined with an inverted microscope at a magnification of $40 \times$. The presence or absence of mobile promastigotes was recorded in each well. The final titer was the last dilution for which the well contained at least one parasite. The number of parasite per gram was calculated in the following way: Parasite burden $=-\log _{10}$ (parasite dilution/ tissue weight)(Rafati et al. 2006a, b; Buffet et al. 1995).

\section{Statistical analysis}

One-way ANOVA statistical test was used to assess the significance of the differences among various groups and Post Hoc LSD test was used to compare the means of different treatment groups. Results with $P<0.05$ were considered to be statistically significant.

\section{Results}

Lymphocyte proliferation assay

To assess the immune-potency of designed nano-vaccine, $\mathrm{BALB} / \mathrm{c}$ mice intramuscularly received 3 injection of the vaccine with simultaneous electroporation pulses followed by an injection of recombinant protein. Analysis of lymphocyte proliferation using Brdu method indicated that after three injection of DNA vaccine and nano-vaccine (before booster injection) proliferation responses of lymphocytes markedly were increased in immunized mice with pcTSA + PMMA, which was significantly higher level of proliferation activity as compared to the control $(P<0.001)$ and DNA vaccine groups $(P<0.014)$. After booster injection no significant difference between vaccinated groups (pcTSA + PMMA and pcTSA) was observed $(P=0.337)$ but the difference of proliferative activity between test $(\mathrm{T})$ and control (C) groups was statistically significant $(P<0.001)$. After challenge with L. major, no significant difference between immunization groups was observed $(P<0.549)$, but both vaccinated groups induced high level of proliferation responses as compared to the control groups $(P<0.001)$ (Fig. 1).

\section{IL-4 and IFN- $\gamma$ cytokine pattern}

In another attempt to evaluate the pattern of cytokine secretion due to the vaccination, culture of individual mouse splenocytes was in vitro re-stimulated with recombinant TSA protein, expressed and purified in $E$. coli cells (data not shown). Collected supernatants were monitored for the amount of IFN- $\gamma$ and IL- 4 to determine the type (T helper 1 vs. $\mathrm{T}$ helper 2 ) of induced immune responses. The

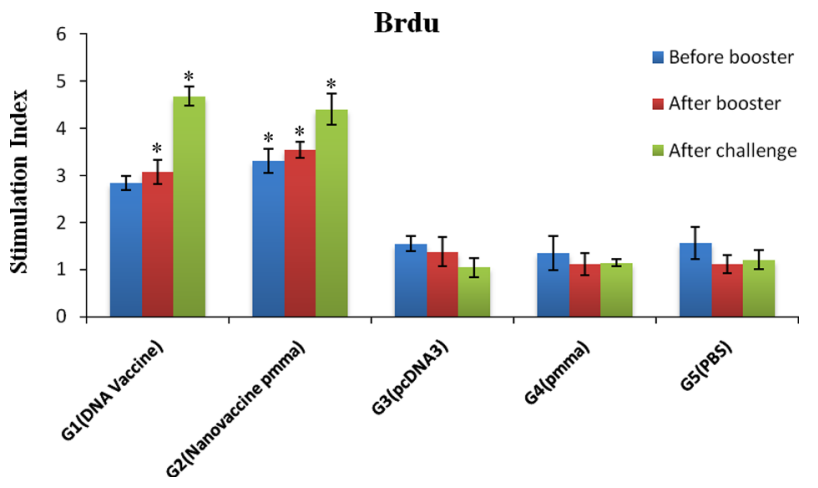

Fig. 1 Lymphocyte proliferation responses following after immunization and after challenge. Mice were immunized with DNA vaccine with or without PMMA ( $n=5$ mice per group) in DNA prime/ peptide boost strategy. Three groups of mice were injected with pcDNA3 vector, PMMA or PBS as negative controls $(n=4$ mice per group). Proliferative responses was done triplicate for individual mice and evaluated with Brdu method as mentioned in Materials and Methods. Data represent mean \pm SD (95 \% C.I.). ${ }^{*} P<0.001$ for pcTSA + PMMA group before peptide booster injection which was significantly higher level of proliferation activity than the other groups. $* P<0.001$ for vaccinated groups compared to all control groups following the booster injection and after challenge

results showed that before booster injection, nano-vaccine induced high level of IFN- $\gamma$ in comparison with DNA vaccine $(P=0.336)$ and control groups $(P<0.005)$, After booster injection IFN- $\gamma$ secretion level in the vaccinated groups was significant difference as compared with control groups $(P<0.001)$ and immunization of mice with DNA vaccine alone increased IFN- $\gamma$ producing lymphocytes as compared with nano-vaccine, though this increase was no significant difference $(P=0.234)$. After challenge level of IFN- $\gamma$ markedly increased in immunized mice with nanovaccine (pcTSA + PMMA), which was significantly higher than DNA vaccine (PcTSA) and control groups $(P<0.001)$ (Fig. 2a). Immunization with DNA vaccine formulated with PMMA nanoparticles showed significant increase in IL-4 producing lymphocytes compared to DNA vaccine alone $(P<0.001)$. After booster injection IL-4 level markedly increased in the group of mice immunized with pcTSA + PMMA, which was significantly higher than PcTSA $(P=0.023)$ and control groups $(P<0.025)$. After challenge with $L$. major no statistically significant difference between PcTSA + PMMA and PcTSA groups was observed $(P>0.733)$ (Fig. 2b).

\section{Antibody response}

Results of total antibodies in the experimental groups show that before recombinant peptide booster injection immunized group with nano-vaccine, significantly increased total antibodies as compared to the control groups $(P<0.003)$. Following the booster injection and after challenge, mice 

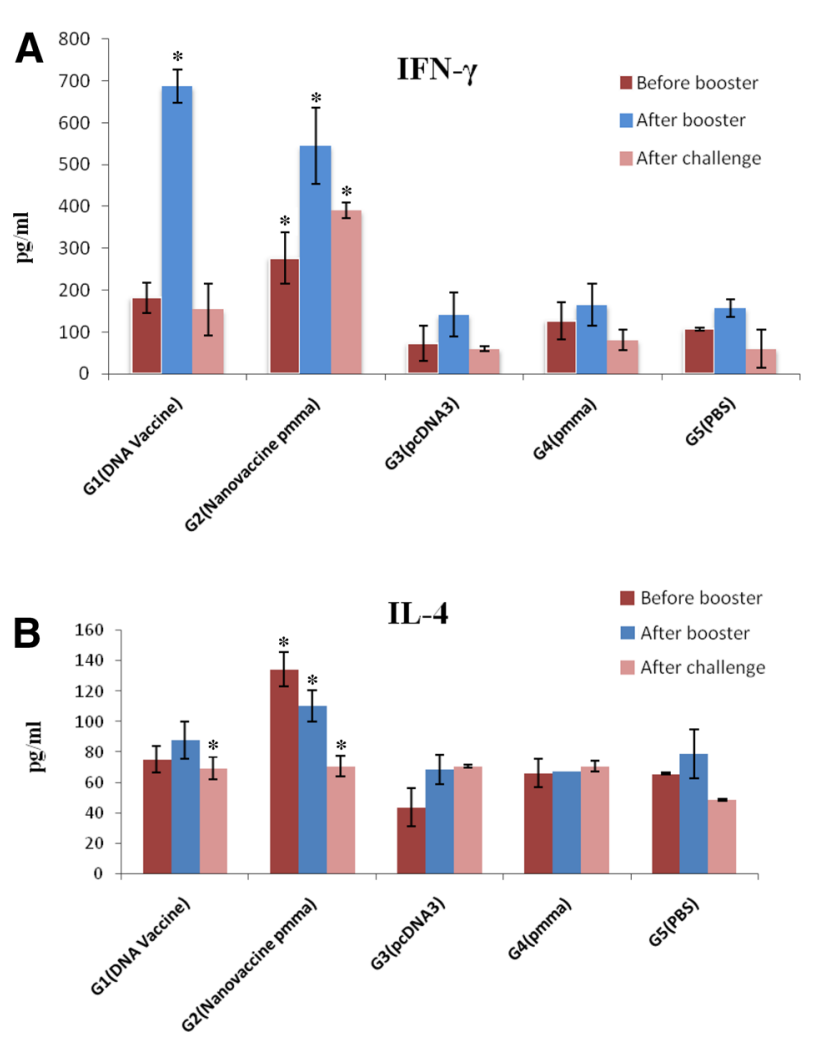

Fig. 2 Cytokine production [IFN- $\gamma(\mathbf{a})$ and IL-4 (b)] by spleen cells of BALB/c mice after immunization periods (before and after booster injection) and after challenge. Mice were immunized with DNA vaccine with or without PMMA ( $n=5$ mice per group) in DNA prime/peptide boost strategy. Three groups of mice were injected with pcDNA3 vector, PMMA or PBS as negative controls $(n=4$ mice per group). Cytokine analyses were monitored using ELISA method during the study as mentioned in materials and methods. Experiments were carried out in duplicate for individual mice. Values represent mean $\pm \mathrm{SD}$ (95 \% C.I.). $* P<0.005$ for nano-vaccine group compared to all other groups before booster injection. ${ }^{*} P<0.001$ for immunized groups compared to the control groups after booster injection. $* P<0.001$ for pcTSA + PMMA group compared to all other groups after challenge with $L$. major (Fig. 2a). $* P<0.025$ for nano-vaccine group compared to all other groups before and after peptide booster injection. ${ }^{*} P<0.011$ for vaccinated groups compared to PBS group after challenge (Fig. 2b)

were immunized with DNA vaccine and boosted with recombinant peptide significantly increased total antibodies as compared to control groups (groups 3, 4 and 5) $(P<0.031)$. Immunization of mice with vaccine candidate formulated with PMMA (group 1) after booster injection and also after challenge showed high level of induction of total antibodies as compared to the negative control groups but no statistical significant difference was observed $(P>0.059)$ (Fig. 3a, b, c).

Results of IgG isotyping showed that following the booster injection IgG1 level in the group of mice which received DNA vaccine was significantly increased compared to nano-vaccine and control groups $(P=0.012$ and $P<0.002$ respectively). Moreover all vaccine immunized groups (groups 1,2 ) significantly increased IgG2a isotype as compared to the control groups (groups 3, 4, 5) $(P<0.030)$ and immunization of mice with DNA vaccine candidate (group 1) significantly increased IgG2a titer as compared to nano-vaccine group (group 2) $(P=0.041)$. After challenge IgG1 levels in vaccinated groups showed increased significantly compared to control groups $(P<0.018)$ and high level of IgG2a in mice were immunized with DNA vaccine/peptide booster in comparison to control groups was observed $(P<0.001)$ (Fig. 3d, e).

Splenic parasite burden

Protection against leishmaniasis was assessed by measurement of parasite burden in the spleen of infected animals. The splenic parasite burden following by immunization and 7 weeks challenge with $L$. major in all experimental groups showed that the number of viable parasites in the spleen was significant difference between vaccinated and unvaccinated groups. Significant difference was observed between two vaccinated groups $(P<0.001)$ and the mice immunized with nano-vaccine (pcTSA + PMMA) showed significantly lowest number of live parasite compared to the other groups $(P<0.001)$ (Fig. 4).

\section{Discussion}

In recent years, significant progress has been made in the identification of vaccine candidates which can induce a protective response against leishmaniasis, but no effective vaccine is available yet. A number of vaccine strategies have been tested, ranging from killed parasites to recombinant antigens or DNA vaccines (Campos-Neto et al. 2001; Mauel 2002; Webb et al. 1996; Ahmed et al. 2004). DNA vaccines effectively engage both MHC-I and MHC-II pathways allowing for the induction of $\mathrm{CD} 8+$ and $\mathrm{CD} 4+\mathrm{T}$ cells whereas antigen present in soluble form, such as recombinant protein, generally induces only antibody responses. DNA vaccines are easy to produce and can induce strong, long lasting and powerful humeral and cellular immunity. Using the prime-boost immunization strategy was a method which was able to affect the quality and quantity of immune responses (Ivory and Chadee 2004; Kwissa et al. 2003; Rafati et al. 2006a, b). Moreover, some approaches such as application of in vivo electroporation that improve the efficacy of DNA vaccine is needed to be considered (Buchan et al. 2005; Miyazaki and Aihara 2002), In the present study a new nano-vaccine containing TSA DNA plasmid was designed and evaluated for it's immunogenicity in BALB/c mice. TSA (thiol-specific antioxidant antigen) is a immune-dominant antigen of 

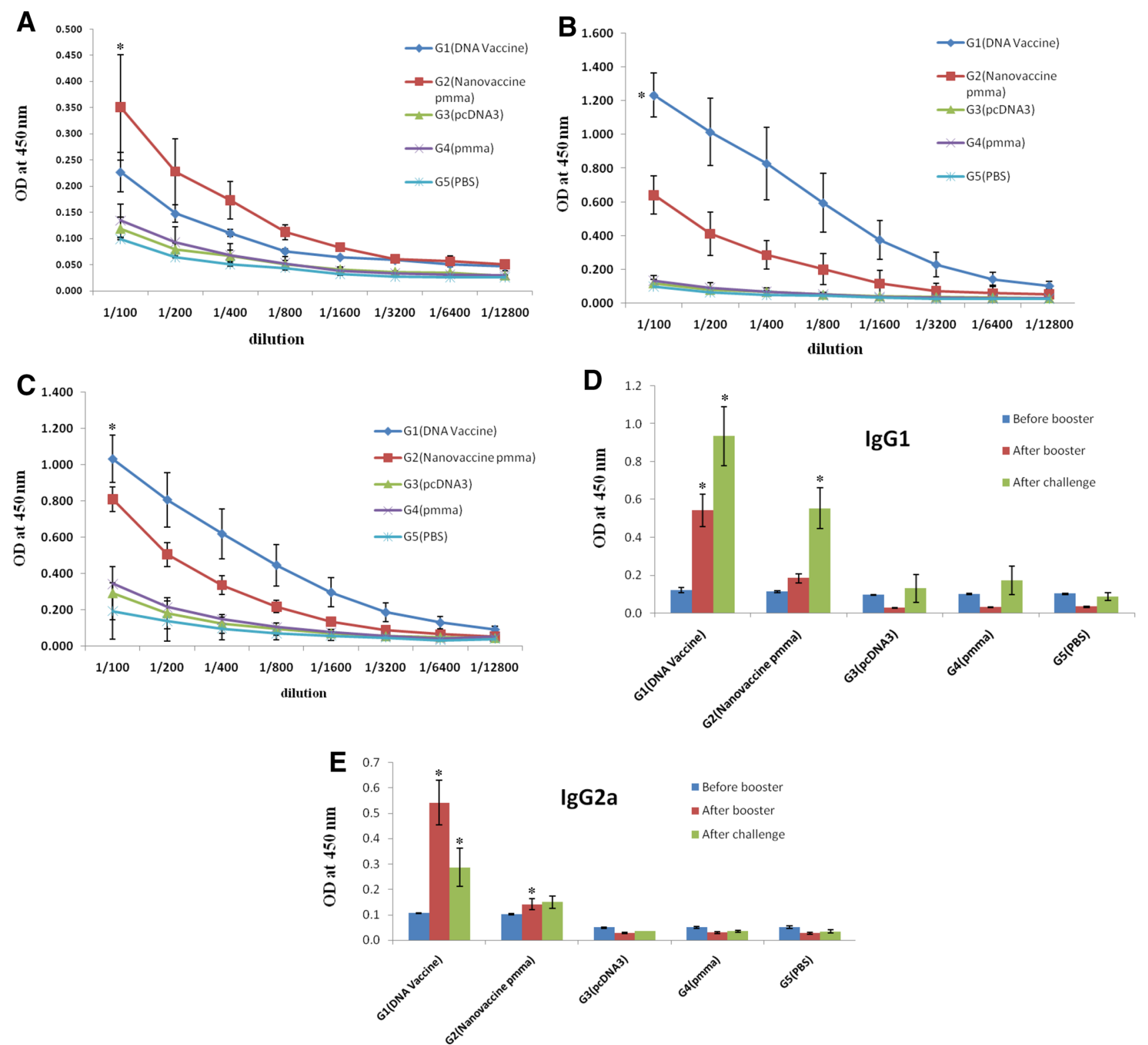

Fig. 3 Specific antibody production against TSA recombinant protein in BALB/c mice immunized with DNA vaccine and nano-vaccine before booster injection (a), following booster injection (b) and after challenge (c) Specific total IgG, IgG1 and IgG2a were measured by ELISA method as mentioned in "Methods" section. Sera from each group were diluted 1:200 and evaluated for the presence of IgG1 and IgG2a. Specific changes of IgG1 levels during the study (d). Specific IgG2a level changes in the study (e). Detection was done with the substrate TMB and optical density at $450 \mathrm{~nm}$ was determined. All data represent mean \pm SD (95\% C.I.). The results indicated the significant difference of total antibody between $\mathrm{G} 2$ and control groups

L. major and is antigenic in both murine and human systems and is constitutive expressed in both promastigote and amastigote life stages (Fatemeh et al. 2012). Immunization of BALB/c mice with a TSA plasmid DNA confers high levels of protective immunity. This DNA vaccine induces CTL activity and solid protection. It also stimulates high after 3 injection of vaccine (i.m) $(* P<0.003)$ (a). After peptide booster injection and after challenge the significant difference of total antibody was observed between $\mathrm{G} 2$ and control groups $(* P<0.048)$ (b, c). $* P<0.041$ for DNA vaccine group compared to all other groups after booster injection $(\mathbf{d}, \mathbf{e}) . * P<0.030$ for vaccinated group compared to control groups following the booster injection (e). $* P<0.018$ for $\mathrm{G} 1$ and $\mathrm{G} 2$ compared to control groups after challenge with $L$. major (d). $* P<0.001$ for DNA vaccine/peptide booster compared to other groups after challenge (e). There was no significant difference among groups before booster injection $(P>0.05)$

titers of specific $\operatorname{IgG} 1, \operatorname{IgG} 2 \mathrm{a}$ antibodies. Further, it also induces a predominant IFN- $\gamma$ production and low levels of IL-4, phenotypic markers of Th1 responses. The recombinant leishmanial antigens LmSTI1 and TSA have been shown that they can induce excellent protection in both murine and nonhuman primate models of human cutaneous 


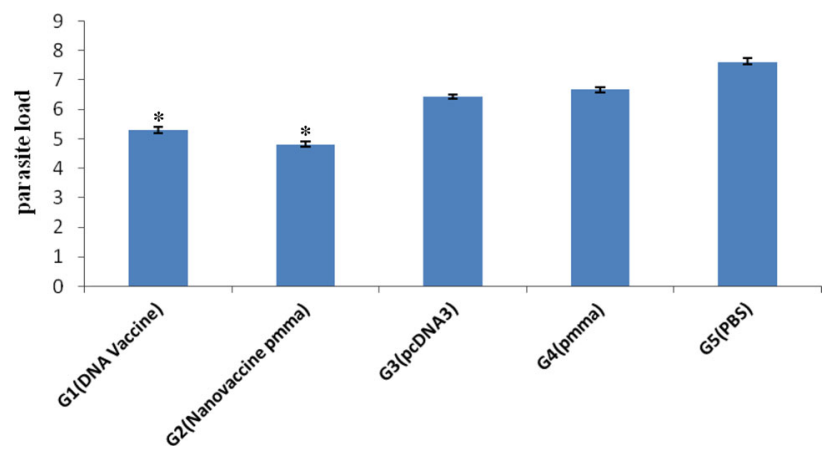

Fig. 4 Parasite burden at 7 weeks after challenge in vaccinated (DNA vaccine, nano-vaccine) in prime/boost strategy and control groups (vector only/PMMA and PBS). The total numbers of viable parasites presented in the spleen of the infected animals were calculated as (parasite dilution per tissue weight). Values represent mean \pm SD for individual mice $(n=3)$. The parasite burden in the spleen of vaccinated mice was significantly less than in the control mice $(* P<0.001)$

leishmaniasis. It seems that the use of an adjuvant and/or delivery system is necessary for almost any modern vaccine particularly vaccines against leishmaniasis (Crowther 2013; Badiee et al. 2013). In this research PMMA nanoparticles was utilized as adjuvant to improve specific humoral and cellular immune responses to our candidate vaccine. PMMA nanoparticle adjuvant achieved good antibody responses and good protection against challenge with a number of antigens. Additionally, they also seem to lead to a higher stability of the vaccines containing this type of adjuvant PMMA nanoparticles are polymeric particulate adjuvant for vaccines. These nanoparticles can easily be manufactured in a reproducible manner in the described particle sizes and with specific surface properties. Among the numerous advantages of the nanoparticles can be pointed simple and easy to produce, process low degradation, safety of adverse effects during use of them. The Use of Poly methylmethacrylate adjuvant for Split Influenza vaccines showed safety record and excellent and stronger protection. In this research the animals were sacrificed and their spleen cells were obtained and cultured in the presence of specific antigen. To evaluate the proliferative responses of lymphocytes Brdu test was used. Brdutest is commonly used in the detection of proliferating cells in tissues (Special living tissues) and is Sensitive, Accurate, Uptodate technique and capable comparative with thymidine radioactive hence Bromodeoxyuridine (BrdU) competes with thymidine (TdR). Results showed that immunization with nano-vaccine formulated with PMMA before and after booster injection induced higher level of lymphocyte proliferation as compared to the DNA vaccine group so nano-formulation of vaccines with PMMA could increase lymphocyte proliferation activity against candidate vaccine. Recombinant TSA protein elicited in vitro proliferative responses from peripheral blood mononuclear cells of human leishmaniasis patients. In the study of Kreuter et al., it was observed that application of HIV-1 Tat antigen formulated with PMMA particles induced proliferation responses of lymphocytes (Kreuter 1996; Kreuter et al. 1976; Voltan et al. 2007). Culture supernatants from stimulated spleen cells were collected and IFN- $\gamma$ and IL-4 concentration were measured by ELISA test. Cytokine analysis of experimental groups revealed that candidate nano-vaccine increased IFN- $\gamma$ cytokine compared to DNA vaccine alone in the immunized groups, before booster injection and after challenge. After booster injection level of IFN- $\gamma$ cytokine in vaccinated groups considerably increased in comparison with before booster injection. Moreover, before and after booster injection IL-4 titer showed increase in the group of mice immunized with nano-vaccine as compare to DNA vaccine alone. These findings amend previous studies by revealing that nanovaccine and prime boost strategy induce Th1 and Th2 pattern of immune responses (Badiee et al. 2013; DaneshBahreini et al. 2011; Rafati et al. 2006a, b). Also, the findings of this study showed that immunization of mice with nano-vaccine or DNA vaccine enhanced total antibody responses as compare with control groups. Before booster injection high level of total antibody was observed in mice immunized with nano-vaccine and following the booster injection and after infection with $L$. major the level of total specific antibody in the group of mice immunized with DNA vaccine was higher than the other vaccinated group. Previous findings demonstrated that PMMA nanoparticle enhanced humoral responses in Hiv-2 Split Whole virus and there was safety of adverse effects during use of them (Stieneker et al. 1995). Other study suggested that PMMA adjuvant may represent an attractive alternative to increase the efficacy of candidate vaccines toward antibody production (Kreuter et al. 1976). While exploring IgG isotypes our results revealed that both specific IgG1 and IgG2a were augmented. Considering that IgG1 is a Th2 marker and IgG2a is a Th1 marker (Campos-Neto 2005), these funding indicated that after peptide booster injection level of $\mathrm{IgG} 1$ and $\operatorname{IgG} 2 \mathrm{a}$ isotypes in immunized groups (nano-vaccine and DNA vaccine) increased. Studies of Campos-Neto et al. showed that Immunization of BALB/c mice with a TSA plasmid DNA induced high titers of specific IgG1, IgG2a antibodies against Leishmania (Campos-Neto et al. 2002). The highest reduction in parasite burden was observed in immunized mice with pcTSA + PMMA and using prime-boost vaccination regimen resulted in enhanced protection against Leishmania infection. The results of previous studies showed that use of nanoparticles (Mutiso et al. 2010; Badiee et al. 2013; Danesh-Bahreini et al. 2011) and prime boost strategy enhanced protective immunity in animal models of 
Leishmania infection (Tewary et al. 2005). In this study, we demonstrated that PMMA can effect on efficacy of a DNA vaccine encoding TSA against $L$. major infection and elicits humoral and cellular immune responses to the antigen delivered. The vaccine formulation described here may be an excellent candidate for further vaccine development against Leishmania.

\section{References}

Ahmed SB, Bahloul C, Robbana C, Askri S, Dellagi K (2004) A comparative evaluation of different DNA vaccine candidates against experimental murine leishmaniasis due to $L$. major. Vaccine 22:1631-1639

Ahmed SB, Touihri L, Chtourou Y, Dellagi K, Bahloul C (2009) DNA based vaccination with a cocktail of plasmids encoding immunodominant Leishmania (Leishmania) major antigens confers full protection in BALB/c mice. Vaccine 27:99-106

Badiee A, Heravi Shargh V, Khamesipour A, Jaafari MR (2013) Micro/nanoparticle adjuvants for antileishmanial vaccines: present and future trends. Vaccine 31:735-749

Buchan S, Gronevik E, Mathiesen I, King CA, Stevenson FK, Rice J (2005) Electroporation as a "prime/boost" strategy for naked DNA vaccination against a tumor antigen. J Immunol 174:6292-6298

Buffet PA, Sulahian A, Garin YJ, Nassar N, Derouin F (1995) Culture microtitration: a sensitive method for quantifying Leishmania infantum in tissues of infected mice. Antimicrob Agents Chemother 39:2167-2168

Campos-Neto A (2005) What about Th1/Th2 in cutaneous leishmaniasis vaccine discovery? Brazilian J Med Biol Res = Revista brasileira de pesquisas medicas e biologicas/Sociedade Brasileira de Biofisica [et al] 38:979-984

Campos-Neto A, Porrozzi R, Greeson K, Coler RN, Webb JR, Seiky YA et al (2001) Protection against cutaneous leishmaniasis induced by recombinant antigens in murine and nonhuman primate models of the human disease. Infect Immun 69:4103-4108

Campos-Neto A, Webb JR, Greeson K, Coler RN, Skeiky YA, Reed SG (2002) Vaccination with plasmid DNA encoding TSA/ LmSTI1 leishmanial fusion proteins confers protection against Leishmania major infection in susceptible BALB/c mice. Infect Immun 70:2828-2836

Crowther JR (2013) ELISA. Theory and practice. Methods Mol Biol 1995(42):1-218

Danesh-Bahreini MA, Shokri J, Samiei A, Kamali-Sarvestani E, Barzegar-Jalali M, Mohammadi-Samani S (2011) Nanovaccine for leishmaniasis: preparation of chitosan nanoparticles containing Leishmania superoxide dismutase and evaluation of its immunogenicity in BALB/c mice. Int J Nanomed 6:835-842

Fatemeh G, Fatemeh T, Zohreh S, Abdolhosein D, Mohammad Zahir H, Mehdi M (2012) Cloning of a recombinant plasmid encoding thiol-specific antioxidant antigen (TSA) gene of Leishmania major and expression in the Chinese hamster ovary cell line. Malays J Med Sci 19:15-19

Gradoni L (2001) An update on antileishmanial vaccine candidates and prospects for a canine Leishmania vaccine. Vet Parasitol 100:87-103

Handman E (2001) Leishmaniasis: current status of vaccine development. Clin Microbiol Rev 14:229-243
Ivory C, Chadee K (2004) DNA vaccines: designing strategies against parasitic infections. Genet Vaccines Ther 2:17

Kreuter J (1995) Nanoparticles as adjuvants for vaccines. Pharm Biotechnol 6:463-472

Kreuter J (1996) Nanoparticles and microparticles for drug and vaccine delivery. J Anat 189(Pt 3):503-505

Kreuter J, Mauler R, Gruschkau H, Speiser PP (1976) The use of new polymethylmethacrylate adjuvants for split influenza vaccines. Exp Cell Biol 44:12-19

Kwissa M, Lindblad EB, Schirmbeck R, Reimann J (2003) Codelivery of a DNA vaccine and a protein vaccine with aluminum phosphate stimulates a potent and multivalent immune response. J Mol Med 81:502-510

Lou PJ, Cheng WF, Chung YC, Cheng CY, Chiu LH, Young TH (2009) PMMA particle-mediated DNA vaccine for cervical cancer. J Biomed Mater Res Part A 88:849-857

Mauel J (2002) Vaccination against Leishmania infections. Curr Drug Targets Immune Endocr Metabol Disord 2:201-226

Mendez S, Gurunathan S, Kamhawi S, Belkaid Y, Moga MA, Skeiky YA et al (2001) The potency and durability of DNA- and protein-based vaccines against Leishmania major evaluated using low-dose, intradermal challenge. $\mathrm{J}$ Immunol 166:5122-5128

Mendez S, Belkaid Y, Seder RA, Sacks D (2002) Optimization of DNA vaccination against cutaneous leishmaniasis. Vaccine 20:3702-3708

Miyazaki J, Aihara H (2002) Gene transfer into muscle by electroporation in vivo. Methods Mol Med 69:49-62

Monnerat S, Martinez-Calvillo S, Worthey E, Myler PJ, Stuart KD, Fasel N (2004) Genomic organization and gene expression in a chromosomal region of Leishmania major. Mol Biochem Parasitol 134:233-243

Mutiso JM, Macharia JC, Gicheru MM (2010) A review of adjuvants for Leishmania vaccine candidates. J Biomed Res 24:16-25

O'Hagan DT (2000) Methods in molecular medicine, vaccine adjuvants: preparation methods and research protocols. Humana Press, Totowa

Rafati S, Salmanian AH, Hashemi K, Schaff C, Belli S, Fasel N (2001) Identification of Leishmania major cysteine proteinases as targets of the immune response in humans. Mol Biochem Parasitol 113:35-43

Rafati S, Zahedifard F, Nazgouee F (2006a) Prime-boost vaccination using cysteine proteinases type I and II of Leishmania infantum confers protective immunity in murine visceral leishmaniasis. Vaccine 24:2169-2175

Rafati S, Ghaemimanesh F, Zahedifard F (2006b) Comparison of potential protection induced by three vaccination strategies (DNA/DNA, Protein/Protein and DNA/Protein) against Leishmania major infection using Signal Peptidase type I in BALB/c mice. Vaccine 24:3290-3297

Saldarriaga OA, Travi BL, Park W, Perez LE, Melby PC (2006) Immunogenicity of a multicomponent DNA vaccine against visceral leishmaniasis in dogs. Vaccine 24:1928-1940

Sambrook J, Fritsch EF, Maniatis T (1989) Molecular cloning: a laboratory manual. Cold Spring Harbor Laboratory Press, New York

Sasaki S, Takeshita F, Xin KQ, Ishii N, Okuda K (2003) Adjuvant formulations and delivery systems for DNA vaccines. Methods 31:243-254

Stieneker F, Kersten G, van Bloois L, Crommelin DJ, Hem SL, Lower $\mathrm{J}$ et al (1995) Comparison of 24 different adjuvants for inactivated HIV-2 split whole virus as antigen in mice. Induction of titres of binding antibodies and toxicity of the formulations. Vaccine 13:45-53

Tabatabaie F, Ghaffarifar F, Sharifi Z, Dalimi A, Zavaran Hoseini A (2007) Cloning and sequencing of Leishmania major thiol- 
specific antioxidant antigen (TSA) gene. Iranian $\mathrm{J}$ Parasitol 2:30-41

Tewary P, Jain M, Sahani MH, Saxena S, Madhubala R (2005) A heterologous prime-boost vaccination regimen using ORFF DNA and recombinant ORFF protein confers protective immunity against experimental visceral leishmaniasis. J Infect Dis 191:2130-2137

Voltan R, Castaldello A, Brocca-Cofano E, Altavilla G, Caputo A, Laus $M$ et al (2007) Preparation and characterization of innovative protein-coated poly(methylmethacrylate) core-shell nanoparticles for vaccine purposes. Pharm Res 24:1870-1882

Webb JR, Kaufmann D, Campos-Neto A, Reed SG (1996) Molecular cloning of a novel protein antigen of Leishmania major that elicits a potent immune response in experimental murine leishmaniasis. J Immunol 157:5034-5041

Webb JR, Campos-Neto A, Ovendale PJ, Martin TI, Stromberg EJ, Badaro R et al (1998) Human and murine immune responses to a novel Leishmania major recombinant protein encoded by members of a multicopy gene family. Infect Immun 66:3279-3289

Zadeh-Vakili A, Taheri T, Taslimi Y, Doustdari F, Salmanian AH, Rafati S (2004) Immunization with the hybrid protein vaccine, consisting of Leishmania major cysteine proteinases Type I (CPB) and Type II (CPA), partially protects against leishmaniasis. Vaccine 22:1930-1940 